\title{
Association of the CCR5 332 variant with juvenile idiopathic arthritis in a meta-analysis
}

\author{
THCM Reinards ${ }^{1 *}$, HM Albers ${ }^{1}$, DMC Brinkman 1 , SSM Kamphuis ${ }^{2}$, MAJ van Rossum³ ${ }^{3}$ EPAH Hoppenreijs ${ }^{4}$, \\ HJ Girschick ${ }^{5}$, C Wouters ${ }^{6}$, RK Saurenmann ${ }^{7}$, JJ Houwing-Duistermaat ${ }^{1}$, REM Toes ${ }^{1}$, TWJ Huizinga ${ }^{1}$, R ten Cate ${ }^{1}$, \\ MW Schilham ${ }^{1}$
}

From 18th Pediatric Rheumatology European Society (PReS) Congress

Bruges, Belgium. 14-18 September 2011

\section{Background}

CCR5 is expressed on Th1 cells and may play a role in Rheumatoid Arthritis by recruiting these cells to the synovium, where they drive an inflammatory process. The $C C R 5 \Delta 32$ variant, a deletion variant which leads to a dysfunctional receptor, has been reported in several genetic association studies in Juvenile Idiopathic Arthritis (JIA), with conflicting results. CCL14 is one of the ligands of CCR5 and polymorphisms in the CCL14 gene have been reported to be associated with Systemic Lupus Erythematosus.

\section{Aim}

We performed a case-control genetic association study to investigate whether CCR5 and CCL14 polymorphisms are associated with susceptibility to JIA.

\section{Methods}

CCR5 32 and CCL14 rs16971802 were genotyped in 667 JIA cases and 1320 healthy controls, both of NorthWest-European white origin. Patients with oligoarticular (persistent and extended), polyarticular (rheumatoid factor negative and positive) and systemic JIA have been included. A meta-analysis combined with three published studies on CCR5 $\triangle 32$ in JIA, with comparable allele frequencies in controls, was performed.

\section{Results}

CCR5 $\triangle 32$ and CCL14 rs16971802 were not significantly associated with JIA in this study, with p-values of 0.12 and 0.72 respectively. Nevertheless, meta-analysis demonstrated association of CCR $5 \Delta 32$ with protection to JIA (combined $\mathrm{p}=0.0003, \mathrm{OR}=0.83,95 \% \mathrm{CI}$ : $0.75-0.91$, Breslow-Day $\mathrm{p}=0.87$, heterogeneity I-squared $=0.0 \%$ ).

\section{Conclusion}

This study has not demonstrated significant associations of CCR5 and CCL14 polymorphisms with JIA, but the association of CCR5 $\triangle 32$ with protection from developing JIA is strengthened in a meta-analysis. It is hypothesized that function of CCR5 could influence synovial inflammation also in JIA.
Author details

${ }^{1}$ Leiden University Medical Center, Leiden, The Netherlands. ${ }^{2}$ Erasmus Medical Center, Rotterdam, The Netherlands. ${ }^{3}$ Academic Medical Center, Amsterdam, The Netherlands. ${ }^{4}$ Radboud University Medical Center, Nijmegen, The Netherlands. 'University of Würzburg, Würzburg, Germany. ${ }^{6}$ University Hospital Gasthuisberg, Leuven, Belgium. 'University Children's Hospital, Zürich, Switzerland.

Published: 14 September 2011

doi:10.1186/1546-0096-9-S1-P287

Cite this article as: Reinards et al:: Association of the CCR5 32 variant with juvenile idiopathic arthritis in a meta-analysis. Pediatric Rheumatology 2011 9(Suppl 1):P287.

* Correspondence: t.h.c.m.reinards@lumc.nl

${ }^{1}$ Leiden University Medical Center, Leiden, The Netherlands

Full list of author information is available at the end of the article

(c) 2011 Reinards et al; licensee BioMed Central Ltd. This is an open access article distributed under the terms of the Creative Commons 